\title{
Undifferentiated Malignant Neoplasm
}

National Cancer Institute

\section{Source}

National Cancer Institute. Undifferentiated Malignant Neoplasm. NCI Thesaurus. Code C36051.

A neoplasm that has minimal to no differentiating features. 